\title{
DECLARACIONES DE MISIÓN DE EMPRESAS EMISORAS EN LA BOLSA DE VALORES Y PRODUCTOS DE ASUNCIÓN, PARAGUAY
}

\author{
MISSION STATEMENTS OF ISSUING COMPANIES ON ASUNCION'S STOCK EXCHANGE, \\ PARAGUAY
}

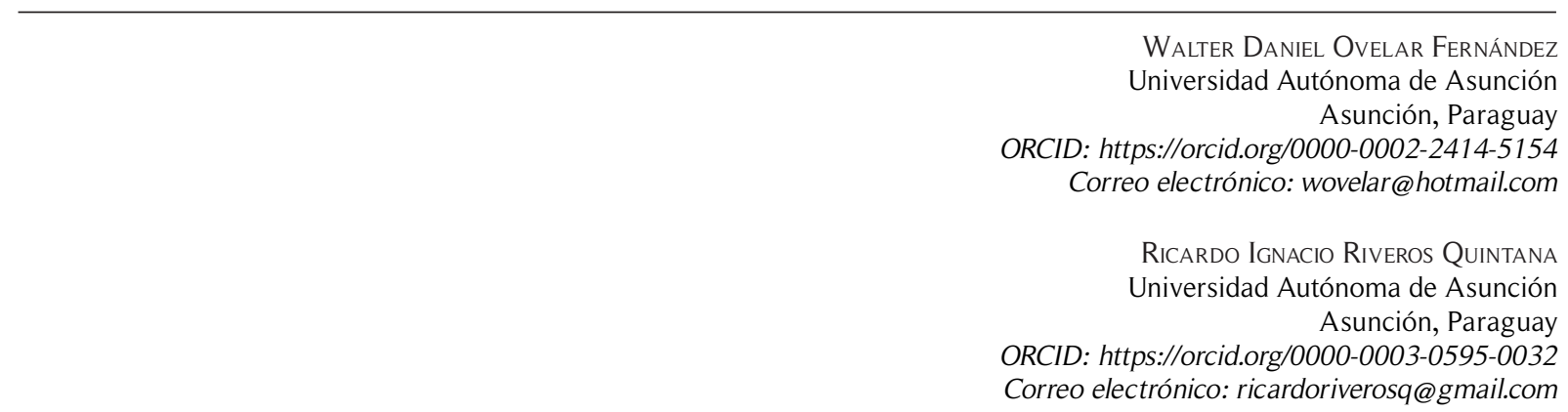

\section{RESUMEN}

Objetivo: Conocer la calidad de las declaraciones de misión de las empresas emisoras de títulos en la Bolsa de Valores y Productos de Asunción Sociedad Anónima (BVPASA), evaluando los componentes y stakeholders (partes interesadas) existentes en las mismas. Método: La investigación fue de tipo cualitativo, obteniéndose las declaraciones de misión directamente de los sitios web de las 30 empresas seleccionadas. Para conocer la calidad de las declaraciones de misión se optó por el modelo de Pearce y David, empleando el análisis de contenido. Dichos autores refieren que, para ser de calidad, una declaración de misión debe reunir nueve componentes principales. Resultados: Fueron dos: a) el promedio de los nueve componentes presentes en las declaraciones de misión asciende al 37\%; y b) la cantidad de stakeholders identificados en las declaraciones de misión es del $24 \%$. Conclusión: Las declaraciones de misión de las empresas analizadas no se adecúan al modelo seleccionado y tampoco mencionan a los stakeholders más importantes; por ende, son de baja calidad.

Palabras clave: Declaración de misión; bolsa de valores; análisis de contenido; stakeholders; calidad.

\begin{abstract}
Objective: To know the quality of the mission statements of the companies issuing securities in the Bolsa de Valores y Productos de Asunción Sociedad Anónima (BVPASA) by evaluating the components and their stakeholders. Method: The research was qualitative, obtaining the mission statements directly from the websites of the 30 selected companies. To know the mission statements' quality, the Pearce and David model was chosen and worked out with content analysis. These authors state that, in order for a mission statement to be of high quality, it must have nine main components. Results: There were two: a) the average of the nine components presented in the mission statements goes up to $37 \%$; and b) the percentage of stakeholders identified in the mission statements was of $24 \%$. Conclusion: The companies' mission statements do not fit the selected model and do not mention the most important stakeholders; therefore, they lack of quality.
\end{abstract}

Keywords: Mission statement; stock exchange; content analysis; stakeholders; quality.

(C) Los autores. Este artículo es publicado por la revista Quipukamayoc de la Facultad de Ciencias Contables, Universidad Nacional Mayor de San Marcos. Este es un artículo de acceso abierto, distribuido bajo los términos de la licencia Creative Commons Atribución 4.0 Internacional (CC BY 4.0) [https://creativecommons. org/licenses/ by/4.0/deed.es] que permite el uso, distribución y reproducción en cualquier medio, siempre que la obra original sea debidamente citada de su fuente original. 


\section{INTRODUCCIÓN}

Una declaración de misión (DM) sería el ikigai japonés, la raison d'être francés o simplemente la razón de ser de la empresa. En este sentido, son varios los académicos que coinciden que una DM es muy importante para el posicionamiento estratégico de cualquier empresa (Rajasekar, 2013). Pearce y David (1987) establecen que la elaboración de una DM es una de las tareas iniciales en el procedimiento de planificación estratégica. Para Cochran, David y Gibson (2008) una DM tal vez sea la parte pública más perceptible del plan estratégico de una empresa. Para Amran (2012), las DM se han convertido en una herramienta básica de negocios, aplicada especialmente para la explicación de las misiones y propósitos de manera clara. De esta manera, las necesidades de desarrollar objetivos realistas y alcanzables han impulsado su popularidad en el sector. Algunas definiciones de DM se exponen en la Tabla 1.

Una DM se caracteriza por tener un alcance extenso, obviar datos monetarios o referirse a porcentajes; por lo general, su extensión no supera unas 250 palabras. Su contenido es inspirador; refiere las utilidades de los productos que ofrece; indica la responsabilidad social que asume; y la responsabilidad ambiental también deberá estar detallada. Los componentes centrales en los que se enfoca son: clientes, productos o servicios, mercados, tecnología, preocupación por la supervivencia, el crecimiento, la rentabilidad, filosofía, autoconcepto, preocupación por la imagen pública, preocupación por los empleados; cuenta con un tono conciliatorio; y posee cualidad de perdurabilidad (David, 2013).

Entre los tantos beneficios proveídos por una DM, Bartkus, Glassman y McAfee (2000) mencionan los siguientes:
1. Comunicar el sentido de la dirección de la empresa y propósito. 2. Servir como mecanismo de control para mantener la empresa "en el buen camino". 3. Ayudar a tomar una amplia gama de decisiones cotidianas. 4. Inspirar y motivar a los empleados.

Sin embargo, también existen detractores de las DM siendo Ackoff (1987), citado por Hofler (2019), uno de ellos, ya que para él son inservibles.

Esta investigación pretende contribuir sobre el estudio crítico de las DM, para la literatura científica latinoamericana en general y a la paraguaya en particular. Con respecto a otros análisis sobre el tema, se han identificado unos pocos estudios en Brasil y uno en el Paraguay.

La BVPASA es la única bolsa de valores operativa en el Paraguay; fue instaurada en el año 1977 y, en la actualidad, es una bolsa de valores $100 \%$ electrónica. Según indican los registros, los valores negociados en el 2019 ascendieron a USD 1026157556.

En concordancia con estudios similares a nivel mundial, las teorías de la señalización y de los stakeholders son la base del porqué una empresa elabora y publica su DM. Justamente los resultados obtenidos por Amran (2012) se soportan en los postulados de la teoría de la señalización, ya que las empresas creen que al estar en una mejor posición deben señalizar esta situación a los inversores para atraer inversiones y mejorar su reputación. Por otro lado, en lo referente a la teoría de los stakeholders, si bien los accionistas (shareholders) son los stakeholders más importantes, Freeman (1994) declara que los objetivos de negocios deben ser conFigurados teniendo también en cuenta los intereses de los otros stakeholders.

Tabla 1

Definiciones de DM

\begin{tabular}{|c|c|c|c|}
\hline $\mathbf{N}^{\circ}$ & Autor/es & Año & Definición \\
\hline 1 & Pearce y David & 1987 & $\begin{array}{l}\text { "Afirmación perdurable acerca del propósito que distingue a una empresa de otras } \\
\text { similares. La declaración de la misión identifica el alcance de las operaciones de una } \\
\text { empresa en términos de producto y mercado" (Pearce y David, 1987, p.1) }\end{array}$ \\
\hline 2 & Charles, Hill y Gareth, Jones, & 2009 & "La misión describe qué hace la compañía" (Hill y Jones, 2009, p.11) \\
\hline 3 & D’Alessio Ipinza Fernando A. & 2008 & $\begin{array}{l}\text { "La misión es el impulsor de la organización hacia la situación futura deseada. Es el } \\
\text { catalizador que permite que esta trayectoria de cambio sea alcanzada por la organi- } \\
\text { zación." (D’Alessio Ipinza, 2008, p. 62) }\end{array}$ \\
\hline 4 & Peter Drucker & 1986 & $\begin{array}{l}\text { "Una institución existe para un propósito y misión específica, que consiste en cumplir } \\
\text { una función social específica. En los negocios esto significa obtener desempeño eco- } \\
\text { nómico" (Drucker, 1986, p. 32) }\end{array}$ \\
\hline
\end{tabular}

Fuente: Elaboración propia con base en Ovelar (2020) 
El objetivo del presente estudio es doble. En primer lugar, se pretende analizar críticamente las DM de las empresas que emiten valores en la BVPASA con el modelo de P\&D (Pearce y David, 1987); y, en segundo lugar, se busca identificar la cantidad de stakeholders claves presentes en estas DM con el fin de determinar si son o no de calidad.

\section{MATERIAL Y MÉTODOS}

Se llevó a cabo un estudio de tipo descriptivo con abordaje cualitativo. Para obtener la cantidad de empresas emisoras se recurrió a la página web de la BVPASA y se utilizó como principal criterio de inclusión a aquellas empresas pertenecientes a los rubros industrial, construcción, comercial o de servicios. Se decidió como criterio de exclusión descartar a las empresas del rubro financiero, entidades públicas y fideicomisos.

Sobre la base de estos criterios, la población seleccionada inicial ascendió a 54 empresas emisoras. Se verificó que cada una de ellas contase con una página web y que sus DM estén publicadas en las mismas. Como resultado, ante la ausencia de páginas web o la no publicación de DM, la muestra seleccionada final fue de 30 empresas. La recolección de los datos se efectuó entre los meses de agosto a octubre del 2020. En la Tabla 2, se listan las empresas emisoras seleccionadas, el rubro al que pertenecen y sus respectivas páginas web.

Tabla 2

Empresas emisoras seleccionadas

\begin{tabular}{|c|c|c|c|c|c|}
\hline $\mathbf{N}^{\circ}$ & Código & Emisor & Rubro & Página web & $\begin{array}{l}\text { Cantidad } \\
\text { de palabras }\end{array}$ \\
\hline 1 & ATL & ATLANTIC S.A.E. & Comercial & https://www.atlantic.com.py/ & 28 \\
\hline 2 & $\mathrm{CHA}$ & CHACOMER S.A.E. & Comercial & www.chacomer.com.py & 21 \\
\hline 3 & DPI & DP INTERNACIONAL S.A.E. & Comercial & https://dpinternacional.com.py/ & 33 \\
\hline 4 & ELE & ELECTROBAN S.A.E.C.A. & Comercial & www.eban.com.py & 18 \\
\hline 5 & IMA & IMAG S.R.L. & Comercial & https://www.imag.com.py/ & 34 \\
\hline 6 & IMC & IMPORT CENTER S.A. & Comercial & https://importcenter.com.py & 39 \\
\hline 7 & INV & INVERFIN S.A.E.C.A. & Comercial & https://inverfin.com.py/ & 6 \\
\hline 8 & KUR & KUROSU \& CIA. S.A. & Comercial & www.kurosu.com.py/» & 18 \\
\hline 9 & LCR & LCR S.A.E.C.A. & Comercial & https://www.lcrsaeca.com/ & 34 \\
\hline 10 & MCT & MERCOTEC S.A.E. & Comercial & http://www.mercotec.com.py/ & 8 \\
\hline 11 & NGO & NICOLAS GONZALEZ ODDONE S.A.E.C.A. & Comercial & http://www.ngosaeca.com.py/ & 26 \\
\hline 12 & NUC & NUCLEO S.A. (PERSONAL) & Comercial & https://www.personal.com.py/ & 6 \\
\hline 13 & PNR & PENNER AUTOMOTORES S.R.L. & Comercial & http://www.grupopenner.com.py/ & 53 \\
\hline 14 & PYC & PROYEC S.A. & Comercial & https://www.proyec.com.py/ & 18 \\
\hline 15 & REC & RECORD ELECTRIC S.A.E.C.A. & Comercial & https://www.recordelectric.com/ & 3 \\
\hline 16 & ROS & ROSANTI S.A.E.C.A. & Comercial & https://www.sls.com.py/ & 42 \\
\hline 17 & MAT & SANITARIOS MATERSAN S.A. & Comercial & http://www.matersan.com.py/ & 18 \\
\hline 18 & TAP & TAPE RUVICHA S.A.E.C.A. & Comercial & https://www.taperuvicha.com/ & 54 \\
\hline 19 & TEL & TELECEL S.A.E. & Comunicación & https://www.tigo.com.py/ & 14 \\
\hline 20 & SWE & SALUM \& WENZ S.A. & Construcción & https://www.salum-wenz.com.py/ & 32 \\
\hline 21 & APC & $\begin{array}{l}\text { ALEMAN PARAGUAYO CANADIENSE S.A. } \\
\text { (ALPACASA) }\end{array}$ & Industrial & https://www.alpacasa.com/ & 31 \\
\hline 22 & BDA & BLUE DESIGN S.A.E. & Industrial & http://www.bdadenim.com/ & 20 \\
\hline 23 & COD & CODIPSA & Industrial & https://www.codipsa.com.py/ & 32 \\
\hline 24 & FRI & FRIGORÍFICO CONCEPCIÓN S.A. & Industrial & https://www.frigorificoconcepcion.com.py/ & 52 \\
\hline 25 & PET & INDUSTRIAS PET S.A.E.C.A. & Industrial & https://www.inpet.com.py/ & 16 \\
\hline 26 & SSP & SUPERSPUMA DEL PARAGUAY S.A.E.C.A. & Industrial & https://www.superspuma.com.py/ & 38 \\
\hline 27 & BEP & BEPSA DEL PARAGUAY S.A.E.C.A. & Servicio & https://www.bepsa.com.py/ & 25 \\
\hline 28 & PAS & PASFIN S.A.E.C.A. & Servicio & https://www.pasfin.com/ & 18 \\
\hline 29 & PTP & PTP PARAGUAY S.A.E. & Servicio & http://www.ptpgroup.com.ar/ & 14 \\
\hline \multirow[t]{3}{*}{30} & DAT & DATA SYSTEMS S.A.E.C.A. & Tecnológico & https://www.data.com.py/ & 18 \\
\hline & & Total de variables utilizadas en las DM & & & 769 \\
\hline & & Promedio de variables utilizadas en las DM & & & 26 \\
\hline
\end{tabular}

Fuente: Elaboración propia 
Luego, se procedió a leer y analizar cada una de las DM utilizando el método del análisis de contenido. El análisis de contenido es "una técnica de investigación destinada a formular, a partir de ciertos datos, inferencias reproducibles y válidas que puedan aplicarse a su contexto" (Krippendorff, 1990, p. 28). Por otra parte, este método de análisis también se aplica "en el ámbito de lo descriptivo, pretendiendo descubrir los componentes básicos de un fenómeno determinado extrayéndolos de un contenido dado" (López Noguero, 2002, p. 168). En dicho análisis, para una mejor incorporación e interpretación de la información, se utilizó la versión de prueba del programa informático de análisis de contenido Altas.ti, a través del cual se han obtenido las palabras clave, dando como resultado una nube de palabras que ha sido empleada en el presente estudio.

El análisis de contenido es constantemente aplicado con el fin de realizar evaluaciones sobre la eficacia y la calidad que poseen las DM. Por citar algunos de ellos: Kemp y Dwyer (2003) en su artículo sobre DM de aerolíneas internacionales; Hocayen-da-Silva, Ferreria y de Castro (2006) para el artículo sobre formulación estratégica en instituciones brasileras; Garg (2014) con su artículo relacionado con las DM de mineras sudafricanas; Albers Araújo, De Souza, y Lunkes (2014) en su artículo sobre el análisis de DM de universidades brasileras; Azizi y Hosseinabadi (2014) en su estudio comparativo sobre DM de empresas de Irán, Turquía, India y EEUU; y Alawneh (2015) en su estudio sobre las DM de los bancos jordanos. Mientras que Aureli (2017) destaca que en las ciencias empresariales y contables el análisis de contenido es de uso común.

Como se mencionó anteriormente, para este trabajo se empleó el modelo P\&D (1987), el cual evalúa la calidad con la que ha sido elaborada una DM, a través de la presencia de nueve componentes principales. A nivel mundial, este supone uno de los métodos más aplicados en el mundo académico; es así como diversos autores han aplicado este modelo en el análisis de DM de las empresas, por citar algunos de ellos: Kemp y Dwyer (2003); Hocayen-da-Silva et al. (2006); Garg (2014); Albers Araújo et al. (2014); Azizi y Hosseinabadi (2014); y Alawneh (2015).

En la Tabla 3, se recurre al trabajo de Garg (2014) para exponer los componentes del modelo P\&D y las variables o palabras que serán buscadas en las DM para su posterior análisis. La lógica de la Tabla 3 para cada uno de los componentes buscados es la siguiente:

Clientes: Los estudios revelan que la preocupación, traducida en el cuidado y el interés por los clientes, es perentoria

Tabla 3

Componentes de una DM de calidad y variables/palabras buscadas

\begin{tabular}{|c|c|}
\hline Componentes de una DM (Modelo P\&D) & Variables y/o palabras buscadas (Garg, 2014) \\
\hline 1. Clientes. ¿Quiénes son los clientes de la empresa? & Clientes, consumidores, benefactores \\
\hline $\begin{array}{l}\text { 2. Productos o servicios. ¿Cuáles son los principales productos o } \\
\text { servicios que ofrece la empresa? }\end{array}$ & Bienes, productos, servicios \\
\hline $\begin{array}{l}\text { 3. Mercados. En términos geográficos, ¿en dónde compite la } \\
\text { empresa? }\end{array}$ & País, nación, región, Paraguay, sector \\
\hline $\begin{array}{l}\text { 4. Tecnología. ¿La empresa está al día desde el punto de vista } \\
\text { tecnológico? }\end{array}$ & $\begin{array}{l}\text { Tecnología, digital, seguridad informática, Internet, páginas web, } \\
\text { redes sociales }\end{array}$ \\
\hline $\begin{array}{l}\text { 5. Preocupación por la supervivencia, el crecimiento y la renta- } \\
\text { bilidad. ¿La empresa está comprometida con el crecimiento y la } \\
\text { solidez financiera? }\end{array}$ & $\begin{array}{l}\text { Creación de valor, crecimiento, utilidad, ganancias, retornos, so- } \\
\text { lides económica, desempeño financiero, resultados financieros, } \\
\text { valor económico añadido }\end{array}$ \\
\hline $\begin{array}{l}\text { 6. Filosofía. ¿Cuáles son las creencias, valores, aspiraciones y } \\
\text { prioridades éticas básicos de la empresa? }\end{array}$ & $\begin{array}{l}\text { Principios, integridad, ética, valores, honestidad, responsabilidad, } \\
\text { armonía, inspiración, motivación, confianza, cultura corporativa }\end{array}$ \\
\hline $\begin{array}{l}\text { 7. Autoconcepto. ¿Cuál es la competencia distintiva o la ventaja } \\
\text { competitiva más importante de la empresa? }\end{array}$ & $\begin{array}{l}\text { Innovación, bajo costo, buenos servicios, calidad elevada, eficien- } \\
\text { cia, eficacia, recursos humanos capacitados y motivados, satis- } \\
\text { facción de los clientes, modernidad, adaptación al cambio, dispo- } \\
\text { nibilidad, agilidad, flexibilidad, liderazgo, compromiso }\end{array}$ \\
\hline $\begin{array}{l}\text { 8. Preocupación por la imagen pública. ¿La empresa responde a } \\
\text { las preocupaciones sociales, comunitarias y ambientales? }\end{array}$ & $\begin{array}{l}\text { Responsabilidad social, responsabilidad ambiental, comunidad, } \\
\text { sociedad, retorno a la sociedad, objetivos sociales, objetivos de } \\
\text { desarrollo sostenible, sostenibilidad, sustentabilidad, inclusividad, } \\
\text { diversidad }\end{array}$ \\
\hline $\begin{array}{l}\text { 9. Preocupación por los empleados. ¿Los empleados son un activo } \\
\text { valioso para la empresa? }\end{array}$ & Empleados, colaboradores, asociados, staff \\
\hline
\end{tabular}

Fuente: Elaboración propia con base en Ovelar (2020), David (2013) y Garg (2014) 
en la formulación de las estrategias, su posterior implementación y, finalmente, al momento de su evaluación (Kemp y

Dwyer, 2003; Albers Araújo et al., 2014).

Servicios: Kemp y Dwyer (2003) postulan que los servicios proporcionados por una empresa deben diferenciarla de sus competidores; prestar atención a este aspecto, clarificándolo en lo posible, es de vital importancia.

Mercados: La palabra mercados debería poder responder a la interrogante: ¿En dónde compite la empresa? (David, 2013). Esto involucra la tarea de detallar la localización geográfica en que la empresa ejerce. Aun así, dicha localización se hace cada vez menos relevante respecto a épocas anteriores a la revolución digital que se vive actualmente (Ovelar, 2020).

Tecnología: Las formas de competir de las empresas han sido modificadas de forma importante en comparación con tiempos pasados, debido a las revoluciones tecnológicas por efecto del Internet y las redes sociales (Ovelar, 2020). El buen uso de la tecnología es uno de los factores que vuelve competitiva a cualquier empresa. Por ejemplo, se requiere que una empresa posea un sitio o una dirección web. De hecho, las DM analizadas en este trabajo fueron colectadas de las respectivas páginas web de las 30 empresas analizadas.

Preocupación por la supervivencia, el crecimiento y la rentabilidad: El leitmotiv de cualquier stakeholders es que las empresas donde poseen cierto tipo de influencia sobrevivan, crezcan y obtengan rentabilidades razonables.
Filosofía: Los autores Kemp y Dwyer (2003) afirman que "si la cultura corporativa se basa en valores, esta se convertirá en una piedra angular (touchstone) para el comportamiento organizacional y la toma de decisiones" (p. 683); es decir, si los valores permean a la organización y los mismos están expuestos en su DM, la misma será exitosa.

Preocupación por la imagen pública: Para Ovelar (2020) el cuidado de lo social y lo ambiental es un imperativo para cualquier organización moderna y, de alguna manera, esta importancia debería reflejarse en su DM.

Preocupación por los empleados: Categóricamente los empleados son stakeholders internos muy importantes para cualquier empresa, pero ¿qué tanta importancia le da una empresa a esta variable/stakeholder en sus DM? Una DM debe usarse como un herramienta de comunicación y, por lo tanto, debe incorporar a los empleados (Höfler, 2019). Para Bartkus et al. (2000) uno de los beneficios de la DM es la de inspirar y motivar a los empleados.

\section{RESULTADOS}

\section{Componentes buscados en las DM analizadas}

En la Tabla 4, se observa que la totalidad de las $30 \mathrm{DM}$ analizadas contienen el 37\% de los componentes buscados.

Se identificó que los componentes con mayores porcentajes fueron: clientes, con el 77\%; servicios, con el $80 \%$; y autoconcepto, con el $80 \%$. Por otro lado, el componente con el menor porcentaje fue tecnología con el $7 \%$.

Tabla 4

Componentes de las DM de las empresas emisoras paraguayas

\begin{tabular}{|c|c|c|c|c|c|c|c|c|c|c|c|c|}
\hline $\mathbf{N}^{\circ}$ & Emisores & a & b & c & d & e & f & g & h & $\mathbf{i}$ & Totales & Porcentajes \\
\hline 1 & ATLANTIC S.A.E. & 1 & 1 & 0 & 0 & 0 & 1 & 1 & 0 & 1 & 5 & $56 \%$ \\
\hline 2 & CHACOMER S.A.E. & 1 & 1 & 0 & 0 & 1 & 1 & 1 & 0 & 0 & 5 & $56 \%$ \\
\hline 3 & DP INTERNACIONAL S.A.E. & 1 & 1 & 0 & 0 & 0 & 0 & 1 & 0 & 0 & 3 & $33 \%$ \\
\hline 4 & ELECTROBAN S.A.E.C.A. & 1 & 0 & 1 & 0 & 0 & 0 & 1 & 0 & 0 & 3 & $33 \%$ \\
\hline 5 & IMAG S.R.L. & 1 & 1 & 0 & 0 & 0 & 0 & 1 & 0 & 0 & 3 & $33 \%$ \\
\hline 6 & IMPORT CENTER S.A. & 1 & 0 & 0 & 0 & 0 & 1 & 1 & 0 & 0 & 3 & $33 \%$ \\
\hline 7 & INVERFIN S.A.E.C.A. & 1 & 0 & 0 & 0 & 0 & 0 & 1 & 0 & 0 & 2 & $22 \%$ \\
\hline 8 & KUROSU \& CIA. S.A. & 1 & 1 & 0 & 0 & 1 & 0 & 1 & 0 & 1 & 5 & $56 \%$ \\
\hline 9 & LCR S.A.E.C.A. & 1 & 1 & 0 & 0 & 0 & 0 & 1 & 0 & 0 & 3 & $33 \%$ \\
\hline 10 & MERCOTEC S.A.E. & 0 & 1 & 0 & 0 & 0 & 0 & 0 & 0 & 0 & 1 & $11 \%$ \\
\hline 11 & NICOLAS GONZALEZ ODDONE S.A.E.C.A. & 0 & 1 & 0 & 0 & 0 & 1 & 1 & 0 & 1 & 4 & $44 \%$ \\
\hline 12 & NUCLEO S.A. (PERSONAL) & 1 & 0 & 0 & 0 & 0 & 1 & 0 & 0 & 0 & 2 & $22 \%$ \\
\hline 13 & PENNER AUTOMOTORES S.R.L. & 1 & 1 & 0 & 0 & 0 & 1 & 1 & 0 & 0 & 4 & $44 \%$ \\
\hline 14 & PROYEC S.A. & 0 & 1 & 1 & 0 & 0 & 0 & 1 & 0 & 0 & 3 & $33 \%$ \\
\hline 15 & RECORD ELECTRIC S.A.E.C.A. & 0 & 0 & 0 & 0 & 0 & 0 & 1 & 0 & 0 & 1 & $11 \%$ \\
\hline 16 & ROSANTI S.A.E.C.A. & 1 & 1 & 0 & 0 & 0 & 0 & 1 & 1 & 0 & 4 & $44 \%$ \\
\hline
\end{tabular}


Tabla 4. Continuación

\begin{tabular}{|c|c|c|c|c|c|c|c|c|c|c|c|c|}
\hline $\mathbf{N}^{\circ}$ & Emisores & a & b & c & d & $\mathbf{e}$ & $f$ & g & $\mathbf{h}$ & $\mathbf{i}$ & Totales & Porcentajes \\
\hline 17 & SANITARIOS MATERSAN S.A. & 1 & 0 & 0 & 0 & 0 & 0 & 1 & 0 & 0 & 2 & $22 \%$ \\
\hline 18 & TAPE RUVICHA S.A.E.C.A. & 1 & 1 & 0 & 0 & 1 & 1 & 0 & 1 & 1 & 6 & $67 \%$ \\
\hline 19 & TELECEL S.A.E. & 0 & 1 & 0 & 1 & 0 & 0 & 0 & 0 & 0 & 2 & $22 \%$ \\
\hline 20 & SALUM \& WENZ S.A. & 0 & 1 & 0 & 0 & 1 & 0 & 1 & 0 & 0 & 3 & $33 \%$ \\
\hline 21 & $\begin{array}{l}\text { ALEMAN PARAGUAYO CANADIENSE S.A. } \\
\text { (ALPACASA) }\end{array}$ & 1 & 1 & 0 & 0 & 0 & 0 & 1 & 1 & 0 & 4 & $44 \%$ \\
\hline 22 & BLUE DESIGN S.A.E. & 1 & 1 & 0 & 0 & 0 & 0 & 1 & 0 & 0 & 3 & $33 \%$ \\
\hline 23 & CODIPSA & 1 & 1 & 1 & 0 & 1 & 0 & 0 & 0 & 0 & 4 & $44 \%$ \\
\hline 24 & FRIGORÍFICO CONCEPCIÓN S.A. & 1 & 1 & 0 & 0 & 0 & 0 & 1 & 1 & 0 & 4 & $44 \%$ \\
\hline 25 & INDUSTRIAS PET S.A.E.C.A. & 1 & 1 & 0 & 0 & 0 & 0 & 1 & 0 & 0 & 3 & $33 \%$ \\
\hline 26 & SUPERSPUMA DEL PARAGUAY S.A.E.C.A. & 1 & 1 & 0 & 0 & 0 & 0 & 1 & 0 & 1 & 4 & $44 \%$ \\
\hline 27 & BEPSA DEL PARAGUAY S.A.E.C.A. & 1 & 1 & 1 & 0 & 0 & 0 & 0 & 0 & 0 & 3 & $33 \%$ \\
\hline 28 & PASFIN S.A.E.C.A. & 1 & 1 & 0 & 0 & 0 & 0 & 1 & 0 & 0 & 3 & $33 \%$ \\
\hline 29 & PTP PARAGUAY S.A.E. & 0 & 1 & 1 & 0 & 0 & 0 & 1 & 0 & 0 & 3 & $33 \%$ \\
\hline \multirow[t]{3}{*}{30} & DATA SYSTEMS S.A.E.C.A. & 1 & 1 & 0 & 1 & 0 & 0 & 1 & 0 & 0 & 4 & $0 \%$ \\
\hline & Totales & 23 & 24 & 5 & 2 & 5 & 7 & 24 & 4 & 5 & 99 & \\
\hline & Porcentajes & $77 \%$ & $80 \%$ & $17 \%$ & $7 \%$ & $17 \%$ & $23 \%$ & $80 \%$ & $13 \%$ & $17 \%$ & $37 \%$ & \\
\hline
\end{tabular}

Nota: a. Clientes; b. Servicios; c. Mercados; d. Tecnología; e. Preocupación por la Supervivencia, el Crecimiento y la Rentabilidad; f. Filosofía; g. Autoconcepto; h. Preocupación por la Imagen Pública; i. Preocupación por los Empleados (Ovelar, 2020)

Fuente: Elaboración propia con base en Ovelar (2020)

\section{Comparación de los resultados de este estudio con resul- tados de estudios similares}

La Tabla 5 expone una comparación de los resultados del presente trabajo frente a los obtenidos por otras investigaciones realizadas por: 1) Ovelar (2020) para bancos paraguayos; 2) Mussoi, Lunkes y Valverde da Silva (2013) para empresas que cotizan en la bolsa de valores de San Paulo; y 3) Kemp y Dwyer (2003) para aerolíneas internacionales.

El resultado obtenido fue del $37 \%$ para las DM analizadas versus el 52\%, 44\% y $48 \%$ para los estudios similares.

\section{Frecuencia de las palabras utilizadas}

En la Tabla 6, se consolidó el total de las palabras presentes en las DM.

Se observa que la palabra con mayor presencia es cliente/s con 16 ocurrencias, seguida por calidad con 10, productos con 11 y servicios con 10 .

\section{Nube de palabras}

En la elaboración de la Figura 1, se aplicó el uso de la versión demo del programa informático de análisis de contenido Atlas.ti. Las $30 \mathrm{DM}$ fueron cargadas al programa mencionado y se obtuvo la presente nube de palabras, en la que prevalecen las palabras clientes y calidad con 12 menciones cada una.

\section{Promedio de palabras utilizadas y comparativa con otros estudios}

En la Tabla 7, se obtuvo el promedio de las palabras utilizadas en las DM de las empresas emisoras paraguayas.

El promedio de palabras se obtiene dividiendo el total de variables procedente de la Tabla 2, que asciende a 769, por la cantidad de $30 \mathrm{DM}$. El resultado del promedio es de 26 palabras para las DM analizadas

\section{Cantidad de stakeholders identificados}

En la Tabla 8, se identifica la cantidad de cinco stakeholders claves presentes en las $30 \mathrm{DM}$ analizadas en este trabajo. En línea con la teoría de los stakeholders, es transcendental que una DM incorpore, por lo menos, a los más importantes.

El resultado obtenido es del $24 \%$, es decir, solamente se obtuvieron 36 menciones de stakeholders de 150 posibles. En otras palabras, de las 30 DM analizadas, tan solo el 33\% menciona a dos o más stakeholders. El stakeholder prevaleciente es clientes con 23/30, mientras que los resultados para los demás stakeholders son muy bajos. 
Tabla 5

Comparación de los resultados de este estudio con resultados de estudios similares

\begin{tabular}{|c|c|c|c|c|}
\hline Autores & Ovelar y Riveros $^{1 /}$ & Ovelar Fernández & Mussoi et al. & Kemp y Dwyer \\
\hline Año & 2021 & 2020 & 2011 & 2003 \\
\hline País/Muestra & $\begin{array}{c}\text { Paraguay/30 } \\
\text { BVPASA }\end{array}$ & $\begin{array}{c}\text { Paraguay/11 } \\
\text { Bancos }\end{array}$ & $\begin{array}{l}\text { Brasil/140 } \\
\text { Empresas } \\
\text { BOVESPA }\end{array}$ & $\begin{array}{c}\text { Australia/50 } \\
\text { Aerolíneas } \\
\text { Internacionales }\end{array}$ \\
\hline \multicolumn{5}{|l|}{ Frecuencia de componentes encontrados } \\
\hline Clientes & $77 \%$ & $73 \%$ & $96 \%$ & $72 \%$ \\
\hline Servicios & $80 \%$ & $82 \%$ & $44 \%$ & $32 \%$ \\
\hline Mercados & $17 \%$ & $45 \%$ & $15 \%$ & $42 \%$ \\
\hline Tecnología & $7 \%$ & $27 \%$ & $7 \%$ & $22 \%$ \\
\hline $\begin{array}{l}\text { Preocupación por la Supervivencia, el Crecimiento } \\
\text { y la Rentabilidad }\end{array}$ & $17 \%$ & $27 \%$ & $58 \%$ & $48 \%$ \\
\hline Filosofía & $23 \%$ & $73 \%$ & $56 \%$ & $80 \%$ \\
\hline Autoconcepto & $80 \%$ & $91 \%$ & $30 \%$ & $88 \%$ \\
\hline Preocupación por la Imagen Pública & $13 \%$ & $27 \%$ & $94 \%$ & $30 \%$ \\
\hline Preocupación por los Empleados & $17 \%$ & $27 \%$ & $0 \%$ & $22 \%$ \\
\hline Promedio & $37 \%$ & $52 \%$ & $44 \%$ & $48 \%$ \\
\hline
\end{tabular}

Nota: ${ }^{1 / E s t u d i o ~ a c t u a l ~}$

Fuente: Elaboración propia

Tabla 6

Frecuencia de palabras presentes en las DM

\begin{tabular}{lclclclc}
\hline Palabras & Total & Palabras & Total & Palabras & Total & Palabras & Total \\
\hline cliente/s & 16 & necesidades & 4 & carne & 2 & producción & 2 \\
calidad & 12 & satisfacción & 4 & confiables & 2 & productores & 2 \\
productos & 11 & experiencia & 3 & crecimiento & 2 & rentabilidad & 2 \\
servicios & 10 & integrales & 3 & económico & 2 & respeto & 2 \\
empresa & 7 & país & 3 & empresas & 2 & seguridad & 2 \\
desarrollo & 6 & personas & 3 & excelencia & 2 & trabajo & 2 \\
servicio & 6 & vida & 3 & humano & 2 & tradición & 2 \\
soluciones & 6 & automotores & 2 & innovadores & 2 & transporte & 614 \\
ambiente & 4 & bienestar & 2 & instituciones & 2 & otras & \\
colaboradores & 4 & capacitados & 2 & mercado & 2 & & 2 \\
medio & 4 & capital & 2 & procesos & & \\
\hline
\end{tabular}

Fuente: Elaboración propia con base en Ovelar (2020)

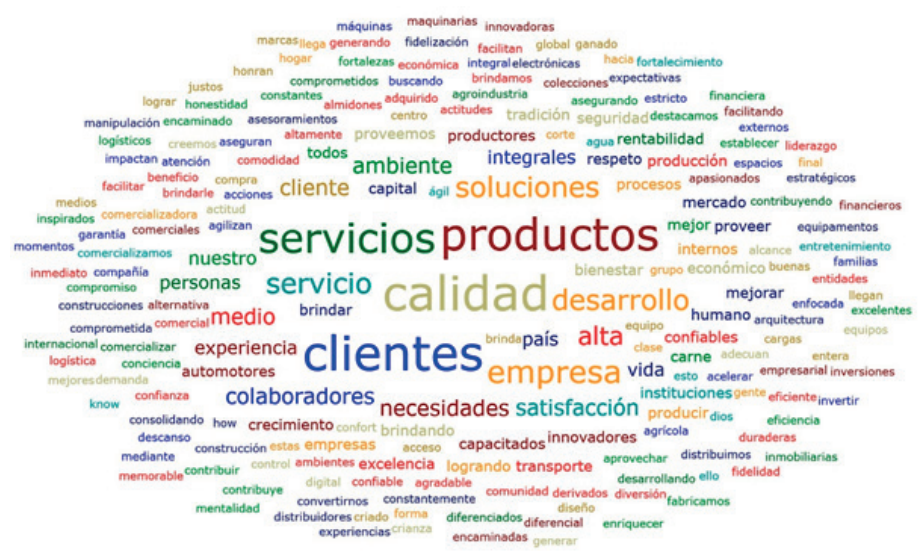

Figura 1. Nube de palabras

Fuente: Elaboración propia 
Tabla 7

Promedio de palabras utilizadas en las DM

\begin{tabular}{lcccc}
\hline & Ovelar y Riveros (2021) & Ovelar (2020) & De Araujo y Nóbrega (2015) & $\begin{array}{c}\text { Ossanes, De Souza } \\
\text { Michelon y Lunkes } \\
\text { (2015) }\end{array}$ \\
\hline Promedio de palabras & 26 & 23 & 24,9 & 22 \\
\hline
\end{tabular}

Nota: ${ }^{1 / E s t u d i o ~ a c t u a l ~}$

Fuente: Elaboración propia

Tabla 8

Suma de stakeholders identificados en las DM

\begin{tabular}{|c|c|c|c|c|c|c|c|}
\hline $\mathbf{N}^{\circ}$ & Emisor & Clientes & Empleados & $\begin{array}{l}\text { Accionistas/ } \\
\text { Inversores }\end{array}$ & Proveedores & Comunidad & Totales \\
\hline 1 & ATLANTIC S.A.E. & 1 & 1 & 0 & 0 & 0 & 2 \\
\hline 2 & CHACOMER S.A.E. & 1 & 0 & 0 & 0 & 0 & 1 \\
\hline 3 & DP INTERNACIONAL S.A.E. & 1 & 0 & 0 & 0 & 1 & 2 \\
\hline 4 & ELECTROBAN S.A.E.C.A. & 1 & 0 & 0 & 0 & 0 & 1 \\
\hline 5 & IMAG S.R.L. & 1 & 0 & 0 & 0 & 1 & 2 \\
\hline 6 & IMPORT CENTER S.A. & 1 & 0 & 0 & 0 & 0 & 1 \\
\hline 7 & INVERFIN S.A.E.C.A. & 1 & 0 & 0 & 0 & 0 & 1 \\
\hline 8 & KUROSU \& CIA. S.A. & 1 & 1 & 0 & 0 & 0 & 2 \\
\hline 9 & LCR S.A.E.C.A. & 0 & 0 & 0 & 0 & 0 & 0 \\
\hline 10 & MERCOTEC S.A.E. & 1 & 0 & 0 & 0 & 0 & 1 \\
\hline 11 & NICOLAS GONZALEZ ODDONE S.A.E.C.A. & 1 & 1 & 0 & 1 & 0 & 3 \\
\hline 12 & NUCLEO S.A. (PERSONAL) & 1 & 0 & 0 & 0 & 0 & 1 \\
\hline 13 & PENNER AUTOMOTORES S.R.L. & 1 & 0 & 0 & 0 & 0 & 1 \\
\hline 14 & PROYEC S.A. & 0 & 1 & 0 & 0 & 0 & 1 \\
\hline 15 & RECORD ELECTRIC S.A.E.C.A. & 0 & 0 & 0 & 0 & 0 & 0 \\
\hline 16 & ROSANTI S.A.E.C.A. & 1 & 0 & 0 & 0 & 1 & 2 \\
\hline 17 & SANITARIOS MATERSAN S.A. & 0 & 0 & 0 & 0 & 0 & 0 \\
\hline 18 & TAPE RUVICHA S.A.E.C.A. & 1 & 1 & 0 & 0 & 0 & 2 \\
\hline 19 & TELECEL S.A.E. & 0 & 0 & 0 & 0 & 0 & 0 \\
\hline 20 & SALUM \& WENZ S.A. & 0 & 0 & 0 & 0 & 0 & 0 \\
\hline 21 & $\begin{array}{l}\text { ALEMAN PARAGUAYO CANADIENSE S.A. } \\
\text { (ALPACASA) }\end{array}$ & 1 & 0 & 0 & 0 & 0 & 1 \\
\hline 22 & BLUE DESIGN S.A.E. & 1 & 0 & 0 & 0 & 0 & 1 \\
\hline 23 & CODIPSA & 0 & 0 & 0 & 1 & 0 & 1 \\
\hline 24 & FRIGORÍFICO CONCEPCIÓN S.A. & 1 & 1 & 0 & 0 & 0 & 2 \\
\hline 25 & INDUSTRIAS PET S.A.E.C.A. & 1 & 0 & 0 & 0 & 0 & 1 \\
\hline 26 & SUPERSPUMA DEL PARAGUAY S.A.E.C.A. & 1 & 1 & 0 & 0 & 0 & 2 \\
\hline 27 & BEPSA DEL PARAGUAY S.A.E.C.A. & 1 & 0 & 0 & 1 & 0 & 2 \\
\hline 28 & PASFIN S.A.E.C.A. & 1 & 0 & 0 & 0 & 0 & 1 \\
\hline 29 & PTP PARAGUAY S.A.E. & 1 & 0 & 0 & 0 & 0 & 1 \\
\hline \multirow[t]{2}{*}{30} & DATA SYSTEMS S.A.E.C.A. & 1 & 0 & 0 & 0 & 0 & 1 \\
\hline & Totales & 23 & 7 & 0 & 3 & 3 & 36 \\
\hline
\end{tabular}

Fuente: Elaboración propia con base en Ovelar (2020) 


\section{DISCUSIÓN}

A continuación, se discute y compara los resultados obtenidos de las Tablas 4 y 5 con relación a los 9 componentes sustanciales de toda DM. Los clientes para Hofler (2019) son stakeholders claves y, por lo tanto, deben jugar un rol importante en cualquier DM. La afirmación anterior se cumple en este trabajo, al observarse en la Tabla 4 que la variable clientes está presente en $77 \%$ de las DM analizadas. Este resultado es cercano al $73 \%$ y al $72 \%$ obtenidos por Ovelar (2020), y Kemp y Dwyer (2003), respectivamente, como se ve en la Tabla 5. El componente servicios, conforme a la Tabla 4, está incorporada en el 80\% de las declaraciones de misión analizadas. Los resultados de este estudio y el $82 \%$ obtenido por Ovelar (2020) para los bancos paraguayos son prácticamente el doble de los resultados obtenidos por Musssoi et al. (2011) y Kemp y Dwyer (2003), según se observa en la Tabla 5. Para mercados, según la Tabla 4, solamente el $17 \%$ de las empresas analizadas incorporan esta variable a sus DM. Este efecto es menor a los que Mussoi et at. (2011) han obtenido con el 33\%; así también al 42\% de Kemp y Dwyer (2003). La tecnología es la variable más olvidada por las empresas paraguayas. El resultado del 7\% es similar al 7\% obtenido por Mussoi et. al. (2011) para las empresas emisoras en el mercado de valores de San Paulo (Tabla 5). En relación con la preocupación por la supervivencia, el crecimiento y la rentabilidad, en este estudio solamente $17 \%$ de las empresas estudiadas mencionan estas variables claves en sus DM, en coincidencia con los resultados de Maragno, De Souza y Lunkes (2013). Este bajo resultado perjudica los intereses de importantes stakeholders, como los accionistas. Este resultado se encuentra por debajo del $58 \%$ y del $48 \%$ presentes en las investigaciones de Mussoi et al. (2011) y Kemp y Dwyer (2003). En cuanto a la filosofía, las DM estudiadas han incorporado variables relacionadas directamente con este componente en un $23 \%$. Al observarse la Tabla 5, este resultado está muy lejos de los obtenidos por los demás estudios utilizados. Con relación al autoconcepto, se ha obtenido como resultado que el $80 \%$ de las empresas aluden en sus DM algunas variables conexas a este componente. El resultado obtenido es cercano al 91\% de Ovelar (2020) para los bancos paraguayos, y al 88\% de Kemp y Dwyer (2003) para las líneas aéreas del mundo. El $13 \%$ obtenido para el componente preocupación por la imagen pública expone la baja preocupación de las empresas estudiadas por su imagen pública. Este resultado es cercano al 8\% obtenido por Maragno et al. (2013), mientras que está alejado de los resultados de Ovelar (2020), con el 27\%. En relación con la preocupación por los empleados, el bajo resultado obtenido por este componente, que asciende al 17\%, no es sorprendente porque es muy cercano a los resultados obtenidos por Ovelar (2020) y Kemp y Dwyer (2003) con el $27 \%$ y el $22 \%$, respectivamente.
Los resultados de la Tabla 6 y la Figura 1 están en línea con los estudios de De Quadros Martins, Diehl y Brunozi Júnior (2016) al ser la palabra clientes la principal "con 159 ocurrencias en las declaraciones de misión de 364 empresas de Argentina, Brasil, Chile, México y Perú" (Ovelar, 2020, p. 8).

Con un promedio de 26 palabras, el resultado de la Tabla 7 está en línea con los resultados que han obtenido anteriormente los estudios de Ovelar (2020), De Araujo y Nobrega (2015) y Ossanes et al. (2015).

Luego, que en la Tabla 8 las 30 DM solamente mencionen al $24 \%$ de los stakeholder claves pone de manifiesto la baja calidad de las DM de las empresas analizadas. $\mathrm{Cu}-$ riosamente, a pesar de que las empresas cuyas DM ha sido analizadas cotizan en una bolsa de valores con el fin de captar inversionistas, en ninguna de ellas se menciona a estos stakeholders claves. El stakeholder clave prevaleciente en este trabajo es clientes al igual que en los estudios de Garg (2014) con 17/58 y Lugoboni y Goto (2016) con $232 / 451$.

En conclusión, y con base en los objetivos establecidos de analizar críticamente las DM con el modelo de Pearce y David (1987) e identificar la cantidad de stakeholders claves presentes en estas DM para determinar si son o no de calidad, la investigación presenta que las DM de las 30 empresas listadas en la BVPASA son de baja calidad, ya que incorporan solamente el $37 \%$ de los componentes principales del modelo de Pearce y David (1987), y mencionan a tan solo el $24 \%$ de sus stakeholders claves. Estos resultados denotan la gran oportunidad de mejora existente para elaborar DM que cumplan con el modelo P\&D (1987) y que mencionen a los stakeholders claves. Como limitaciones, se menciona la baja cantidad de muestras seleccionadas y que corresponden solamente a empresas que emiten títulos en la única bolsa de valores del Paraguay.

En cuanto a la agenda futura de investigación, se plantean las siguientes preguntas: ¿Sería válida la aplicación de encuestas a stakeholders para determinar si las DM son importantes o no?, y ¿existen incidencias de causa-efecto entre la calidad de las DM y la rentabilidad de las empresas investigadas?

\section{REFERENCIAS}

Alawneh, A. (2015). The impact of mission statement on performance: An exploratory study in the Jordanian Banking Industry. Journal of Management Policy and Practice, 16(4), 73-87.

Albers Araújo, M., De Souza, P., \& Lunkes, R. (2014). Análise dos principais elementos de Pearce II (1982) presentes nas missoes organizacionais das Universidades 
Públicas do Brasil. XIV Coloquio Internacional de Gestao Universitaria - CIGU, 1-15.

Amran, N. (2012). Mission Statement and Company Performance: Evidence from Malaysia. International Journal of Business and Behavioral Sciences, 2(4), 98-107.

Araujo, F., \& Nóbrega, K. (2015). Análise comparativa sobre declarações de Missão em empresas de serviços. XI Congresso Nacional de Excelência em Gestão, 1-14.

Aureli, S. (2017). A comparison of content analysis usage and text mining in CSR corporate disclosure. The International Journal of Digital Accounting Research, (17), 1-32. http://doi.org/10.4192/1577-8517-v17_1

Azizi S., \& Hosseinabadi, V. (2014). A Content Analysis of the Mission Statements of Iran, Turkey, India, and United States Pharmaceutical Companies. Management \& Marketing, XII (1).

Bartkus, B., Glassman, M., \& McAfee, R. B. (2000). Mission statements: Are they smoke and mirrors? Business Horizons, 43(6), 23-28. https://doi.org/10.1016/s00076813(00)80018-x

Cochran, D. S.; David, F. R., \& Gibson, C. K. (2008). A framework for developing an effective mission statement. Journal of Business Strategies, 25(2), 27-39.

D’Alessio Ipinza, F. (2008). El proceso estratégico. Un enfoque de gerencia. México D.F.: Pearson Educación de México.

David, F. R. (2013). Conceptos de Administración Estratégica (14ta ed.). México D.F.: Pearson Educación de México.

De Quadros Martins, V., Diehl, C., \& Brunozi Júnior, A. (2016). Análise da efetividade das missões empresarias e sua relação com fatores contingenciais em países do GLENIF. XXIII Congresso Brasileiro de Custos, 1-16.

Drucker, P. (1986). Management. Tasks, Responsibilities, Practices. New York: Truman Talley Books / E.P. Dutton.

Freeman, R.E. (1994). The politics of stakeholder theory: Some future directions. Business Ethics Quarterly, 4(4), 409-421.

Garg, A. K. (2014). Content analysis of mission statement of mining companies in South Africa. Problems and Perspectives in Management, 12(4).

Hocayen-da-Silva, A., Ferreria, J., \& de Castro, M. (2006). Missão organizacional como instrumento para a formulação de estratégias e obtenção de vantagem com- petitiva: análise comparativa em instituições financeiras brasileiras. Revista de Economia e Administração, 5(2), 221-242.

Hofler, K. (2019). The Mission Statements of Austrian Companies: A comparative content analysis (Tesis de maestría). Lauder Business School, Austria.

Hill, C. W., \& Jones, G. R. (2009). Administración Estratégica. México D.F.: Mc Graw Gill.

Kemp, S., \& Dwyer, L. (2003). Mission statements of international airlines: a content analysis. Tourism Management, 24(6), 635-653. http://doi.org/10.1016/S02615177(03)00049-9

Krippendorff, K. (1990). Metodología de análisis de contenido. Teoría y Práctica. Barcelona: Paidós Comunicación.

López Noguero, F. (2002). El análisis de contenido como método de investigación. Revista de Educación, 4, 167179.

Lugoboni, L., \& Goto, M. (2016). Stakeholders Present in Organizational Mission: an Analysis by Sector and Source of Shareholder Control. Revista de Administração da UFSM, 11(3), 615-636. https://doi. org/10.5902/1983465916171

Maragno, C., De Souza, P., \& Lunkes, R. (2013). Missao Institutional: Analise dos Principais Elementos Presentes nas Missoes dos Hoteis de Santa Catarina. Estudios y Perspectivas en Turismo, 23(3), 467-483.

Mussoi, A., Lunkes, R., \& Valverde da Silva, R. (2011). Missao Institucional: uma Analise da Efetividade e dos Elementos Presentes nas Missoes de Empresas Brasileiras de Capital Aberto. REGE, 18(3), 361-384.

Ossanes, B., De Souza Michelon P., \& Lunkes, R. (2017). Análise dos principais elementos presentes nas missões de hospitais brasileiros de excelencia. Revista Salud Pública, 19(4), 542-548.

Ovelar, W. (2020). Análisis comparativo de las declaraciones de misión de los Bancos que operan en el Paraguay. ACADEMO, 7(1),1-10. http://dx.doi.org/10.30545/ academo.2020.ene-jun.1

Pearce, J., \& David, F. (1987). Corporate mission statements: The bottom line. Academy of management Executive, 1(2), 109-116.

Rajasekar, J. (2013). A Comparative Analysis of Mission Statement Content and Readability. Journal of Management Policy and Practice, 14(6), 131-147. 